6-1-1993

\title{
Thermally Activated Escape Over Fluctuating Barriers
}

Ulrich Zürcher

Cleveland State University, u.zurcher@csuohio.edu

Charles R. Doering

Follow this and additional works at: https://engagedscholarship.csuohio.edu/sciphysics_facpub

Part of the Physics Commons

How does access to this work benefit you? Let us know!

Publisher's Statement

(C)1993 American Physical Society

\section{Repository Citation}

Zürcher, Ulrich and Doering, Charles R., "Thermally Activated Escape Over Fluctuating Barriers" (1993).

Physics Faculty Publications. 280.

https://engagedscholarship.csuohio.edu/sciphysics_facpub/280

This Article is brought to you for free and open access by the Physics Department at EngagedScholarship@CSU. It has been accepted for inclusion in Physics Faculty Publications by an authorized administrator of

EngagedScholarship@CSU. For more information, please contact library.es@csuohio.edu. 


\title{
Thermally activated escape over fluctuating barriers
}

\author{
U. Zürcher* and Charles R. Doering \\ Department of Physics, Clarkson University, Potsdam, New York 13699-5820
}

(Received 30 October 1992)

\begin{abstract}
We investigate the thermally activated escape of a Brownian particle over a potential barrier whose height fluctuates with a rate $\alpha$ between the values $E_{+}$and $E_{-}$. We are mainly interested in the lowtemperature behavior where $E_{+} / T \gg E_{-} / T$. We calculate the mean exit time as a function of the rate of the barrier fluctuations for the piecewise linear and the piecewise constant barrier, $\tau=\tau(\alpha)$. For the piecewise constant potential we find three different regimes: $\tau \sim \tau_{+}$for $\alpha<\tau_{+}^{-1}=\exp \left(-E_{+} / T\right), \tau \sim 2 \tau_{-}$ for $\alpha>\tau_{-}^{-1}=\exp \left(-E_{-} / T\right)$, and $\tau \sim \alpha^{-1}$ for $\tau_{+}^{-1}<\alpha<\tau_{--}^{-1}$. The mean exit time for the piecewise linear potential has a different behavior for fast barrier fluctuations, $\alpha>\tau_{-}^{-1} ; \tau(\alpha)$ is a monotonously increasing function that approaches the asymptotic value $\tau \sim \sqrt{\tau_{+} \tau_{-}}$for $\alpha \rightarrow \infty$. We show that the behavior of the mean exit time for the piecewise constant potential is characterized by the absence of correlations between barrier crossings and barrier fluctuations. We discuss these correlations in some detail for the piecewise linear potential barrier.
\end{abstract}

PACS number(s): 05.40. $+\mathrm{j}, 02.50 .-\mathrm{r}, 82.20 . \mathrm{Mj}$

\section{INTRODUCTION}

The diffusion of a particle over a barrier has attracted much attention over the past 50 years. It has many applications in problems from physics, chemistry, biology, and other fields. A particle is moving in a potential with two local equilibrium positions that are separated by a potential barrier. The particle is coupled to a heat bath so that in a reduced description, the particle is subject to both a damping force and a fluctuating random force. In many cases the motion of the Brownian particle is approximated by a Markov process whose transition probabilities obey a Fokker-Planck equation [1].

The particle stays near one of its equilibrium positions for a long time until the particle jumps to the top of the barrier due to rare energy fluctuations. The escape rate is governed by a Boltzmann factor, $w \propto \exp (-\Delta E / T)$, where $\Delta E$ is the potential difference between the top and the bottom of the potential and $T$ is the temperature of the heat bath (in units with $k_{B}=1$ ). The prefactor is determined by the properties of the motion of the particle near the bottom and the top of the barrier as well as the strength of the coupling between the particle and the heat bath. An account of the exhaustive discussion of these questions is found in a recent review [2].

In the last decade, thermally activated escape has been studied in systems in which other dynamic processes are present. In particular, nonlinear cooperative effects have been observed in systems with fluctuational transitions and periodic modulations both experimentally and theoretically. It was found that the addition of external noise may amplify the signal-to-noise ratio (SNR) in a periodically driven bistable system (stochastic resonance) [3-8].

The standard model for stochastic resonance is an overdamped Brownian particle which is moving in a bistable potential and is driven periodically. At low-noise level, the particle hops infrequently between the wells with a rate that increases with increasing noise level. In the intermediate-noise-level regime, the likelihood of a hop is greatest when the deterministic modulation reaches its maximum value. With further increase of the noise level, the particle hops frequently between the wells. The SNR thus has a characteristic domelike shape.

In this paper, we study the thermally activated escape of a Brownian particle over a fluctuating barrier $[9,10]$. In our model, we take for the barrier height a dichotomic random process. That is, the barrier height fluctuates with a rate $\alpha$ between the values $E_{+}$and $E_{-}$. This model has previously been studied by one of us [11] and this work constitutes a more complete presentation and expansion of that work. It was found that as a function of the rate of the barrier fluctuations, the mean exit time has a resonancelike behavior.

We may expect that at all times, the Brownian particle escapes over either the large or the small barrier. With this assumption, a particular behavior of the mean exit time already follows. For slow barrier fluctuations, the mean exit time is governed by the escape over the large barrier $E_{+}, \tau \sim \tau_{+}=\exp \left(E_{+} / T\right)$, while the escape over the small barrier $E_{-}$governs the mean exit time for fast barrier fluctuations, $\tau \sim \tau_{-}=\exp \left(E_{-} / T\right)$. In an intermediate regime, the mean exit time is inversely proportional to the rate of the barrier fluctuations, $\tau \sim \alpha^{-1}$. However, in the case of the piecewise linear potential barrier, the Brownian particle is moving in some average potential for fast barrier fluctuations. For the mean exit time, we therefore find $\tau \sim \sqrt{\tau_{+} \tau_{-}}=\exp \left[\left(E_{+}\right.\right.$ $\left.\left.+E_{-}\right) / 2 T\right]$ in the limit $\alpha \rightarrow \infty$. These considerations show that barrier crossings and barrier fluctuations are correlated for a general form of the potential barrier.

The outline of the paper is as follows. In Sec. II, we specify more completely the model of an overdamped Brownian particle moving in a metastable potential with a fluctuating barrier. We find exact results for the mean 
exit time for both the piecewise constant and the piecewise linear potential. The results are discussed in Sec. III. In Sec. IV, we introduce a truncated version of our model that is described by two coupled rate equations. The discussion shows the correlations between barrier crossings and barrier fluctuations. Finally, we summarize and discuss our main results in Sec. $\mathrm{V}$.

\section{MODEL}

The equation of motion of an overdamped Brownian particle is given by

$$
\dot{x}(t)+U^{\prime}(x)=\zeta(t)
$$

where $x(t)$ is the coordinate of the particle at time $t$, $U^{\prime}(x)$ is the deterministic force, and $\zeta(t)$ is the Gaussian noise of zero mean intensity $T$ (in units with $k_{B}=1$ ),

$$
\begin{aligned}
& \langle\zeta(t)\rangle=0, \\
& \langle\xi(t) \xi(s)\rangle=2 T \delta(t-s) .
\end{aligned}
$$

Closed expressions for the diffusion in a bistable potential has been obtained for simple models [12]. We consider the piecewise linear potential,

$$
U_{l}(x)= \begin{cases}(\varepsilon / L)(L-x), & 0 \leq x \leq L, \\ (\varepsilon / L)(L+x), & -L \leq x \leq 0,\end{cases}
$$

and the piecewise constant potential,

$$
U_{c}(x)= \begin{cases}0, & a \leq x \leq L, \\ \varepsilon, & -a \leq x \leq a \\ 0, & -L \leq x \leq-a .\end{cases}
$$

The stochastic process $x(t)$, cf. Eq. (2.1), is defined once we specify the boundary conditions at $x=L$ and $-L$.

We consider the case when the barrier height flips with equal rates between the two values $E_{+}$and $E_{-}$. That is, the barrier height $\varepsilon$ is a Markovian two-state process,

$$
\begin{aligned}
& \varepsilon=\frac{E_{+}+E_{-}}{2}+\frac{E_{+}-E_{-}}{2} \chi(t), \\
& \chi(t)= \pm 1,
\end{aligned}
$$

with equal jump rates $(1 \rightarrow-1)=\alpha$ and $(-1 \rightarrow 1)=\alpha$, respectively. The fluctuating potentials are sketched in Figs. 1(a) and 1(b). The symmetric dichotomic noise has zero mean and an exponential correlation,

$$
\begin{aligned}
& \langle\chi(t)\rangle=0, \\
& \langle\chi(t) \chi(s)\rangle=e^{-2 \alpha|t-s|},
\end{aligned}
$$

Because $(x, \chi)$ is a two-variable Markov process, the mean exit time is obtained in a standard manner [13].
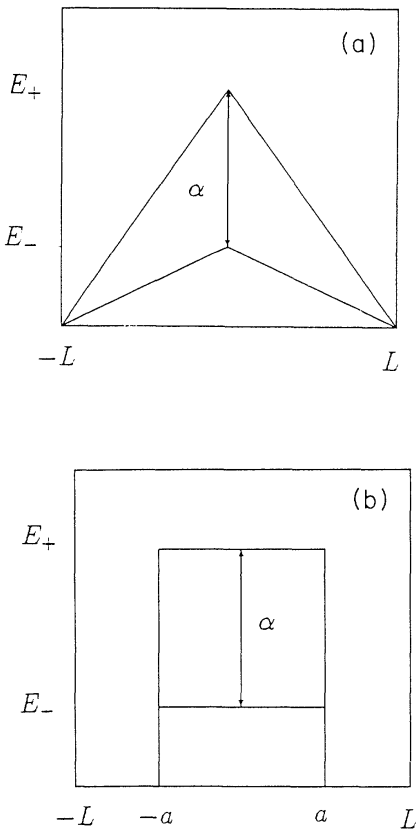

FIG. 1. (a) Sketch of the piecewise linear potential barrier $U_{l}(x)$. (b) Sketch of the piecewise constant potential barrier $U_{c}(x)$.

We define the quantities $\tau_{ \pm}(x)$ by

$$
\left(\begin{array}{cc}
\mathcal{L}_{+}^{\dagger}-\alpha & \alpha \\
\alpha & \mathcal{L}_{-}^{\dagger}-\alpha
\end{array}\right)\left(\begin{array}{l}
\tau_{+} \\
\tau_{-}
\end{array}\right)=-\left(\begin{array}{c}
\frac{1}{2} \\
\frac{1}{2}
\end{array}\right),
$$

where $\mathcal{L}_{ \pm}^{\dagger}$ are the adjoint Fokker-Planck operators for the barriers with the heights $E_{ \pm}$[14]. In Eq. (2.10) we assume the stationary distribution of the barrier height when the diffusion process starts off.

The boundary conditions (BC) for the $\tau_{+}$and $\tau_{-}$must be specified. We choose reflecting $\mathrm{BC}$ at the bottom of the barrier $x=-L$ and absorbing $\mathrm{BC}$ at the top of the barrier $x=0$,

$$
\begin{aligned}
& \frac{d \tau_{ \pm}}{d x}(x=-L)=0, \\
& \tau_{ \pm}(x=0)=0 .
\end{aligned}
$$

With this choice $\tau_{+}(x)$ and $\tau_{-}(x)$ are completely specified. In the subsequent discussion, we are mainly interested in the mean exit time of the Brownian particle that is placed at the bottom of the barrier $x=-L$,

$$
\tau=\tau_{+}(-L)+\tau_{-}(-L) .
$$

The calculation of $\tau_{ \pm}(x)$ is straightforward and is outlined here for the piecewise linear potential $U_{L}(x)$ only. The adjoint Fokker-Planck operators are given by 
$\mathcal{L}_{ \pm}^{\dagger}=-\left(d U_{ \pm} / d x\right)(d / d x)+T\left(d^{2} / d x^{2}\right)$. From Eq. (2.10), we find

$$
\begin{aligned}
& -\frac{E_{+}}{L} \frac{d \tau_{+}}{d x}+\frac{d^{2} \tau_{+}}{d x^{2}}-\alpha \tau_{+}(x)+\alpha \tau_{-}(x)=-\frac{1}{2} \\
& -\frac{E_{-}}{L} \frac{d \tau_{-}}{d x}+T \frac{d^{2} \tau_{-}}{d x^{2}}-\alpha \tau_{-}(x)+\alpha \tau_{+}(x)=-\frac{1}{2} .
\end{aligned}
$$

We introduce

$$
\begin{aligned}
& E=\frac{E_{+}+E_{-}}{2}, \\
& \Delta=\frac{E_{+}-E_{-}}{2},
\end{aligned}
$$

and

$$
\begin{aligned}
& \sigma(x)=\tau_{+}(x)+\tau_{-}(x), \\
& \delta(x)=\tau_{+}(x)-\tau_{-}(x) .
\end{aligned}
$$

We find the coupled differential equations

$$
\begin{aligned}
& -\frac{E}{L} \frac{d \sigma}{d x}+T \frac{d^{2} \sigma}{d x^{2}}+1=\frac{\Delta}{L} \frac{d \delta}{d x}, \\
& -\frac{E}{L} \frac{d \delta}{d x}+T \frac{d^{2} \delta}{d x^{2}}-2 \alpha \delta(x)=\frac{\Delta}{L} \frac{d \sigma}{d x},
\end{aligned}
$$

and the boundary conditions,

$$
\begin{aligned}
& \sigma(x=0)=0, \quad \frac{d \sigma}{d x}(x=-L)=0, \\
& \delta(x=0)=0, \quad \frac{d \delta}{d x}(x=-L)=0 .
\end{aligned}
$$

We introduce a scaled dimensionless coordinate,

$$
x=\frac{L T}{E} y,
$$

so that the length scale of the potential barrier $L$ is given by

$$
y_{0}=\frac{E}{T} .
$$

Three parameters characterize our model,

$$
\begin{aligned}
& \tau_{0}=2 \frac{L^{2} T}{E^{2}}, \\
& \lambda=\alpha \tau_{0}, \\
& \eta=\frac{\Delta}{E} .
\end{aligned}
$$

The Brownian particle is subject to both the stochastic force $\zeta(t)$ and, on the average, the deterministic force $E / L$. Its mean-square displacement increases quadratically in time due to the deterministic force while it increases linearly in time due to the stochastic force. It follows that the motion of the Brownian particle is dominated by the stochastic force on time scales shorter than $\tau_{0}$ while the motion is dominated by the deterministic force on time scales longer than $\tau_{0}$. Thus the time scale $\tau_{0}$ separates the deterministic from the stochastic regime of the Brownian motion in the linear potential. On the time scale $\tau_{0}$, the rate of the barrier fluctuations is given by $\lambda$. Finally, the relative change in the barrier height is given by $\eta$.

The coupled differential equations then read

$$
\begin{aligned}
& -\frac{d \sigma}{d y}+\frac{d^{2} \sigma}{d y^{2}}+\frac{\tau_{0}}{2}=\eta \frac{d \delta}{d y}, \\
& -\frac{d \delta}{d y}+\frac{d^{2} \delta}{d y^{2}}-\lambda \delta(y)=\eta \frac{d \sigma}{d y},
\end{aligned}
$$

and $\sigma(y)$ and $\delta(y)$ are subject to the boundary conditions,

$$
\begin{aligned}
& \frac{d \sigma}{d y}\left(-y_{0}\right)=0, \quad \sigma(0)=0, \\
& \frac{d \delta}{d y}\left(-y_{0}\right)=0, \quad \delta(0)=0 .
\end{aligned}
$$

A straightforward calculation gives the mean exit time $\tau$, cf. Eq. (2.13),

$$
\begin{aligned}
\tau_{l}=\frac{\tau_{0}}{2} D^{-1}[ & -q_{1}\left(q_{1}-1\right)\left(q_{3}-q_{2}\right) e^{-q_{1} y_{0}}+\frac{1}{q_{3}}\left(q_{2}-q_{1}\right)\left(q_{3}-1\right)\left(q_{1} q_{2}+q_{3}^{2}-1\right) e^{-\left(q_{1}+q_{2}\right) y_{0}} \\
& \left.-q_{1} q_{2}\left(q_{2}-q_{1}\right)\left[1+\frac{1}{q_{1} q_{2} q_{3}}-\frac{1}{q_{1}}-\frac{1}{q_{2}}-\frac{1}{q_{3}}\right) e^{-\left(q_{1}+q_{2}+q_{3}\right) y_{0}}+\text { cycl. perm. }\right],
\end{aligned}
$$

where $D$ is given by

$$
D=q_{1} q_{2}\left(q_{2}-q_{1}\right)\left(q_{3}-1\right) e^{-\left(q_{1}+q_{2}\right) y_{0}}+\text { cycl. perm. }
$$

The roots $q_{i}, i=1,2,3$, follow from

$$
\begin{aligned}
& q_{1}+q_{2}+q_{3}=2, \\
& q_{1} q_{2}+q_{2} q_{3}+q_{3} q_{1}=-\left(\lambda+\eta^{2}-1\right), \\
& q_{1} q_{2} q_{3}=-\lambda .
\end{aligned}
$$


We discuss this result in more detail in the next section.

The piecewise constant potential barrier is the limiting case when the Brownian particle is subject to a nonvanishing deterministic force for an infinitesimal time only. In this case, the expectation value of the mean-square displacement between two consecutive changes in the barrier height is a quantity that characterizes the Brownian motion,

$$
\frac{1}{\kappa^{2}}=\frac{T}{2 \alpha}
$$

The mean exit time for the Brownian particle in the fluctuating piecewise constant potential follows:

$$
\begin{aligned}
\tau_{c}=\frac{a(L-a)}{T}[ & \left\{2+\tanh (\kappa a) \tanh [\kappa(L-a)]\left(e^{E_{+} / T}+e^{E_{-} / T}\right)\right\}^{-1} \\
\times & \left(e^{E_{+} / T}\left\{1+\tanh (\kappa a) \tanh [\kappa(L-a)] e^{E_{-} / T}\right\}\right. \\
& \left.\left.+e^{E_{-} / T}\left\{1+\tanh (\kappa a) \tanh [\kappa(L-a)] e^{E_{+} / T}\right\}\right)-1\right]+\frac{L^{2}}{2 T}
\end{aligned}
$$

Thus the mean exit time $\tau_{c}$ depends on the rate of the barrier fluctuations through the combinations $\kappa a$ and $\kappa(L-a)$.

\section{MEAN EXIT TIME}

In the presence of barrier fluctuations, the properties of the mean exit time depend on the rate of the barrier fluctuations on the time scales defined by the thermally activated escape over the small and the large barrier, respectively. Two time scales are thus defined by

$$
\tau_{ \pm}=\exp \left(\frac{E_{ \pm}}{T}\right) \text {. }
$$

The barrier fluctuations are slow for $\alpha<\tau_{+}^{-1}$, while they are fast for $\alpha>\tau_{-}^{-1}$. In the intermediate regime we have $\tau_{+}^{-1}<\alpha<\tau_{-}^{-1}$. In the case of the piecewise linear potential, the mean exit time depends on the rate of the barrier fluctuations through the combination $\lambda=\alpha \tau_{0}$ only, cf. Eqs. (2.27), (2.29), and (2.30). For sufficiently low temperatures or high barriers, we assume $\tau_{+}>\tau_{-}>\tau_{0}$.

We consider first the case of the piecewise linear potential. For $\alpha=0$, the roots $q_{i}, i=1,2,3$, cf. Eqs. $(2.35)-(2.37)$, are given by

$$
\begin{aligned}
& q_{1}=0, \\
& q_{2}=1-\eta, \\
& q_{3}=1+\eta .
\end{aligned}
$$

For $q_{1}=0$, we find from Eqs. (2.33) and (2.34),

$$
\frac{2 D}{\tau_{0}} \tau=q_{2} q_{34}\left(q_{2}-1\right) e^{-q_{2} y_{0}}+\frac{q_{2}}{q_{3}}\left(q_{3}-1\right)\left(q_{3}^{2}-1\right) e^{-q_{3} y_{0}}
$$

and

$$
D=-q_{2} q_{3}\left(q_{3}-q_{2}\right) e^{-\left(q_{2}+q_{3}\right) y_{0}} .
$$

Hence, we find for $\alpha=0$,

$$
\tau_{l}=\frac{\tau_{0}}{4(1+\eta)^{2}} e^{(1+\eta) y_{0}}, \quad \alpha=0
$$

Using $1+\eta=2 E_{+} /\left(E_{+}+E_{-}\right), y_{0}=\left(E_{+}+E_{-}\right) / 2 T$, and $\tau_{0}=8 L^{2} T /\left(E_{+}+E_{-}\right)^{2}$, we have

$$
\tau_{l}=\frac{L^{2} T}{2 E_{+}^{2}} \exp \left(\frac{E_{+}}{T}\right), \quad \alpha=0 .
$$

In the limit $\alpha \rightarrow \infty$, the roots $q_{i}, i=1,2,3$, are given by

$$
\begin{aligned}
& q_{1} \rightarrow-\infty, \\
& q_{2}=\frac{\alpha \tau_{0}}{\alpha \tau_{0}+\eta^{2}} \simeq 1, \\
& q_{3} \rightarrow \infty .
\end{aligned}
$$

It follows that both $D \tau$ and $D$ diverge like $\exp \left(-q_{1} y_{0}\right)$,

$$
\frac{2 D}{\tau_{0}} \tau_{l}=-q_{1}\left(q_{1}-1\right)\left(q_{3}-1\right) e^{-q_{1} y_{0}}
$$

and

$$
D=q_{1}\left(1-q_{1}\right)\left(q_{3}-1\right) e^{-\left(q_{1}+1\right) y_{0}} .
$$

Hence

$$
\tau_{l}=\frac{\tau_{0}}{2} e^{y_{0}}, \quad \alpha \rightarrow \infty,
$$

that is

$$
\tau_{l}=\frac{L^{2} T}{E^{2}} \exp \left[\frac{E_{+}+E_{-}}{2 T}\right], \quad \alpha \rightarrow \infty .
$$

Further, for small $\lambda$, we find in first order,

$$
\begin{aligned}
& q_{1}=-\frac{\alpha \tau_{0}}{1-\eta^{2}} \\
& q_{2}=1-\eta+\frac{\alpha \tau_{0}}{2(1-\eta)}, \\
& q_{3}=1+\eta+\frac{\alpha \tau_{0}}{2(1+\eta)} .
\end{aligned}
$$

Here, we assume $\eta \neq 1$, i.e., $E_{-} \neq 0$. We insert Eqs. (3.16)-(3.18) into Eq. (2.33) and expand its right-hand side (RHS) to first order in $\tau_{ \pm}$and $\alpha \tau_{0}$, 
$\frac{2 D}{\tau_{0}} \tau=-\alpha \tau_{0} \frac{2 \eta}{1-\eta^{2}}-\eta \frac{1-\eta}{1+\eta} \tau_{-}^{-1}-\eta \frac{1+\eta}{1-\eta} \tau_{+}^{-1}$

Similarly, we expand the RHS of Eq. (2.34) to second order in $\tau_{ \pm}^{-1}$ and $\alpha \tau_{0}$,
$D=-\alpha \eta\left(\frac{1-\eta}{1+\eta} \tau_{-}^{-1}+\frac{1+\eta}{1-\eta} \tau_{+}^{-1}\right)-2 \eta(1-\eta) \tau_{+}^{-1} \tau_{-}^{-1}$

Thus, we find

$$
\tau_{l}=\frac{\tau_{0}}{2} \frac{\left(2 E^{2} / E_{+} E_{-}\right) \alpha \tau_{0} \tau_{+} \tau_{-}+\left(E_{-} / E_{+}\right) \tau_{+}+\left(E_{+} / E_{-}\right) \tau_{-}}{\alpha \tau_{0}\left[\left(E_{-} / E_{+}\right) \tau_{+}+\left(E_{+} / E_{-}\right) \tau_{-}\right]+2\left(E_{+} E_{-} / E^{2}\right)}, \quad \tau_{+}^{-1}<\alpha<\tau_{-}^{-1}
$$

We summarize the behavior of the mean exit time over the fluctuating linear potential as a function of the rate of the barrier fluctuations. For slow barrier fluctuations, $\alpha<\tau_{+}^{-1}, \tau_{l}$ is independent of $\alpha$ and $\tau_{l} \simeq \tau_{+}$. The mean exit time then decreases with increasing rate of the barrier fluctuations. We find $\tau_{l} \simeq \alpha^{-1}$ for $\tau_{+}^{-1}<\alpha<\tau_{-}^{-1}$. The mean exit time has a minimum at $\alpha \simeq \tau_{-}^{-1}$ where $\tau_{l} \simeq \tau_{-}$. For fast barrier fluctuations, $\alpha>\tau_{-}^{-1}, \tau_{l}$ increases with increasing rate of the barrier fluctuations and approaches a finite value $\tau_{l} \simeq \sqrt{\tau_{+} \tau_{-}}$as $\alpha \rightarrow \infty$. These three different regimes are clearly seen in Fig. 2. For the numerical calculation we use parameters with $\log _{10} \tau_{+}=14$ and $\log _{10} \tau_{-}=6$.

The closed expression for the mean exit time for the fluctuating piecewise constant potential is given in Eqs. (2.38) and (2.39). For $\alpha=0$, we have $\kappa=0$. We find

$$
\tau_{c}=\frac{a(L-a)}{2 T} \tau_{+}, \quad \alpha=0 .
$$

Similarly, we have $\kappa \rightarrow \infty$ for $\alpha \rightarrow \infty$,

$$
\tau_{c}=2 \frac{a(L-a)}{T} \tau_{-}, \quad \alpha \rightarrow \infty
$$

For intermediate rates of the barrier fluctuations, $\tau_{+}^{-1}<\alpha<\tau_{-}^{-1}$, we assume the length scales of the potential such that $\kappa a<1$ and $\kappa(L-a)<1$. The mean exit time then follows as

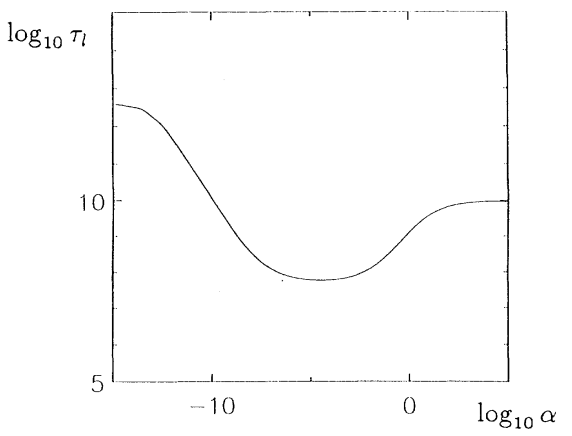

FIG. 2. The mean exit time for the fluctuating piecewise linear potential barrier as a function of the rate of the barrier fluctuations. Parameters are chosen with $\log _{10} \tau_{+}=14$, $\log _{10} \tau_{-}=6$, and $(L-a) a / T=16$.

$$
\begin{gathered}
\tau_{c}=\frac{a(L-a)}{T} \frac{\left[a(L-a) / T^{2}\right] \alpha \tau_{+} \tau_{-}+\tau_{+}+\tau_{-}}{2+2\left[a(L-a) / T^{2}\right] \alpha\left(\tau_{+}+\tau_{-}\right)}, \\
\tau_{+}^{-1}<\alpha<\tau_{-}^{-1} .
\end{gathered}
$$

To summarize, the mean exit time for the fluctuating piecewise constant potential is independent of the rate of the barrier fluctuations for $\alpha<\tau_{+}^{-1}$ and $\tau_{c} \simeq \tau_{+}$. For $\tau_{+}^{-1}<\alpha<\tau_{-}^{-1}$, the mean exit time is inversely proportional to the rate of the barrier fluctuations, $\tau_{c} \simeq \alpha^{-1}$. For fast barrier fluctuations the mean exit time is again independent of $\alpha$ and $\tau_{c} \simeq \tau_{-}$for $\alpha>\tau_{-}^{-1}$. This behavior of $\tau_{c}(\alpha)$ is shown in Fig. 3. Again, we use parameters with $\log _{10} \tau_{+}=14$ and $\log _{10} \tau_{-}=6$.

Thus the mean exit times for the fluctuating piecewise constant and the fluctuating piecewise linear potential have similar behavior for slow barrier fluctuations,

$$
\tau_{c} \sim \tau_{l}, \quad \alpha<\tau_{-}^{-1},
$$

while their behavior is different for fast barrier fluctuations,

$$
\tau_{c} \not \tau_{l}, \quad \alpha>\tau_{-}^{-1} .
$$

For a static potential barrier, the mean exit time is proportional to the thermal activation, $\tau=\tau_{p} \exp (E / T)$. Detailed properties of the potential barrier enter this expression through the prefactor $\tau_{p}$ only. Our results show that in the case of the fluctuating potential, the exponential factor itself depends on details of the potential barrier.

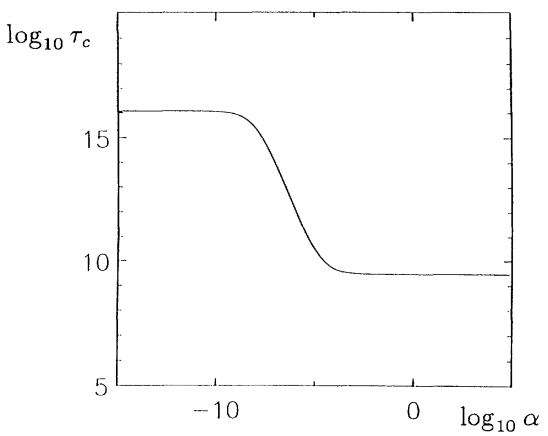

FIG. 3. The mean exit time for the fluctuating piecewise constant potential barrier as a function of the rate of the barrier fluctuations. Parameters are chosen with $\log _{10} \tau_{+}=14$, $\log _{10} \tau_{-}=6$, and $(L-a) a / T=16$. 
This dependence is most clearly seen in the limit of fast barrier fluctuations. In this limit, the Brownian particle moving in the piecewise constant potential escapes with certainty over the small barrier and $\tau_{c} \simeq \tau_{-}$ $=\exp \left(E_{-} / T\right)$ as $\alpha \rightarrow \infty$. In the case of the fluctuating piecewise linear potential, the Brownian particle is subject to the average deterministic force $E / L$ in the limit $\alpha \rightarrow \infty$. The thermal activation then follows as $\tau_{l} \simeq \sqrt{\tau_{+} \tau_{-}}=\exp (E / T)=\exp \left[\left(E_{+}+E_{-}\right) / 2 T\right]$ for $\alpha \rightarrow \infty$.

We conclude that in the presence of barrier fluctuations, thermal activation processes depend on properties of the Brownian motion in the potential well. In the next section, we demonstrate that barrier crossings and barrier fluctuations are correlated depending on detailed properties of the potential barrier.

\section{TRUNCATED MODEL}

For a static potential barrier, we define the probability that at time $t$ the Brownian particle is to the left, say, of the barrier, $n=n(t)$. We assume $n(0)=1$. The particle escapes over the barrier with the rate $r$. We neglect the probability flux of the particle from the right to the left side of the barrier. We find the differential equation for $n(t), d n / d t=-r n(t)$, and hence $n(t)=\exp (-r t)$.

For a barrier whose height fluctuates with a rate $\alpha$ between the two values $E_{+}$and $E_{-}$, we similarly define the joint probabilities $n_{+}(t)$ and $n_{-}(t)$. We assume the equilibrium distribution of the barrier height at the initial time $t=0, n_{+}(0)=n_{-}(0)=\frac{1}{2}$. If the rate of the barrier fluctuations vanishes, the joint probabilities decay independently of each other with the rates $r_{+}=\exp \left(-E_{+} / T\right)$ and $r_{-}=\exp \left(-E_{-} / T\right)$, respectively, i.e., $n_{+}(t)=\frac{1}{2} \exp \left(-r_{+} t\right)$ and $n_{-}=\frac{1}{2} \exp \left(-r_{-} t\right)$. We neglect correlations between barrier crossings and barrier fluctuations and find the coupled rate equations for $n_{+}(t)$ and $n_{-}(t)$ for arbitrary rates of the barrier fluctuations [15],

$$
\begin{aligned}
& \frac{d n_{+}}{d t}=-r_{+} n_{+}(t)-\alpha n_{+}(t)+\alpha n_{-}(t), \\
& \frac{d n_{-}}{d t}=-r_{-} n_{-}(t)-\alpha n_{-}(t)+\alpha n_{+}(t) .
\end{aligned}
$$

From Eqs. (4.1) and (4.2) we derive a second-order differential equation for $n_{+}(t)$. We make the ansatz

$$
n_{+}(t)=e^{-\xi t}, \quad \zeta>0 \text {. }
$$

The decay of the probabilities $n_{ \pm}(t)$ is determined by the smaller of the two roots $r^{*}$. We find

$$
r^{*}=\alpha+\frac{1}{2}\left(r_{+}+r_{-}\right)-\sqrt{\alpha^{2}+\frac{1}{4}\left(r_{-}-r_{+}\right)^{2}} .
$$

For slow and fast barrier fluctuations, respectively (recall that $r_{-}>r_{+}$),

$$
r^{*} \simeq\left\{\begin{array}{l}
r_{+}, \quad \alpha<r_{+}, \\
r_{-} / 2, \quad \alpha>r_{1} .
\end{array}\right.
$$

For intermediate rates, we have

$$
r^{*}=\frac{r_{+} r_{-}+\alpha\left(r_{+}+r_{-}\right)}{2 \alpha+r_{+}+r_{-}}, \quad r_{+}<\alpha<r_{-} .
$$

Or, using $\tau_{ \pm}=r_{ \pm}^{-1}$, we find for the mean exit time $\tau^{*}=1 / r^{*}[16]$,

$$
\tau^{*} \simeq\left\{\begin{array}{c}
\tau_{+}, \quad \alpha<\tau_{+}^{-1}, \\
\left(2 \alpha \tau_{+} \tau_{-}+\tau_{+} \tau_{-}\right) /\left[1+\alpha\left(\tau_{+}+\tau_{-}\right)\right], \\
\tau_{+}^{-1}<\alpha<\tau_{-}^{-1}, \\
2 \tau_{-}^{-1}, \quad \alpha>\tau_{-}^{-1} .
\end{array}\right.
$$

The qualitative behavior of $\tau^{*}(\alpha)$ is identical with the behavior of the mean exit time for the fluctuating piecewise constant potential $\tau_{c}(\alpha)$ over the entire range of the rate of the barrier fluctuations. This result was to be expected. In the case of the piecewise constant potential, the Brownian particle escapes over either the small or the large barrier at all times. Barrier crossings and barrier fluctuations are thus two independent stochastic processes and the behavior of $\tau_{c}$ follows.

In the case of the piecewise linear potential, the time scale $\tau_{0}=2 L^{2} T / E^{2}$ is characteristic for the motion of the Brownian particle inside the potential well. On time scales shorter than $\tau_{0}$, the motion is diffusive (with the diffusion constant $T$ ) while on time scales longer than $\tau_{0}$, the average deterministic force dominates. We introduce joint probabilities that are averaged over the time $\tau_{0}$,

$$
\widetilde{n}_{ \pm}(t)=\frac{1}{\tau_{0}} \int_{t}^{t+\tau_{0}} n_{ \pm}(s) d s
$$

For slow barrier fluctuations, $\alpha \ll \tau_{0}^{-1}$, we have $\widetilde{n}_{ \pm}(t)=n_{ \pm}(t)$ and we recover the model studied above. The probabilities $n_{+}(t)$ and $n_{-}(t)$ are equilibrated on the time scale $\alpha^{-1}$. It follows that for fast barrier fluctuations $\alpha \gg \tau_{0}^{-1}$, the time-averaged probabilities $\widetilde{n}_{+}(t)$ and $\tilde{n}_{-}(t)$ are equal,

$$
\tilde{n}_{+}(t) \simeq \tilde{n}_{-}(t) \simeq \widetilde{n}(t)=\frac{1}{2} e^{-r t}, \quad \alpha \gg \tau_{0}^{-1} .
$$

The average probability $\tilde{n}(t)$ decays in time with the average rate

$$
r=\sqrt{r_{+} r_{-}}=\exp \left(-\frac{E_{+}+E_{-}}{2 T}\right)=\exp \left(-\frac{E}{T}\right) .
$$

For finite values of the rate of the barrier fluctuations, the time-averaged probabilities $\widetilde{n}_{ \pm}(t)$ obey the same rate equations as $n_{ \pm}(t)$ where, however, the bare time constants $r_{ \pm}$and $\alpha$ are replaced by respective renormalized quantities. Since the barrier fluctuates with probability $1-\exp (-\lambda)$ during a time interval of length $\tau_{0}\left(\lambda=\alpha \tau_{0}\right)$, we estimate the renormalized probability fluxes over the barrier as

$$
\begin{aligned}
\widetilde{j}_{ \pm \rightarrow 0} & =-r_{ \pm} e^{-\kappa} \widetilde{n}_{ \pm}(t)-r\left(1-e^{-\kappa}\right) \widetilde{n}_{ \pm}(t), \\
& =-\left[r_{ \pm} e^{-\kappa}+r\left(1-e^{-\kappa}\right)\right] \widetilde{n}_{ \pm}(t), \\
& =-\widetilde{r}_{ \pm} \widetilde{n}_{ \pm}(t)
\end{aligned}
$$


and similarly,

$$
\begin{aligned}
\widetilde{j}_{ \pm \rightarrow \mp} & =-\alpha e^{-\kappa_{n_{ \pm}}(t)} \\
& =-\widetilde{\alpha} \widetilde{n}_{ \pm}(t) .
\end{aligned}
$$

We thus find the rate equations

$$
\begin{aligned}
& \frac{d \widetilde{n}_{+}}{d t}=-\widetilde{r}_{+} \widetilde{n}_{+}(t)-\widetilde{\alpha} \widetilde{n}_{+}(t)+\widetilde{\alpha} \widetilde{n}_{-}(t), \\
& \frac{d \widetilde{n}_{-}}{d t}=-\widetilde{r}_{-} \widetilde{n}_{-}(t)-\widetilde{\alpha} \widetilde{n}_{-}(t)+\widetilde{\alpha} \widetilde{n}_{+}(t),
\end{aligned}
$$

where

$$
\begin{aligned}
& \widetilde{r}_{ \pm}=r_{ \pm} e^{-\kappa}+r\left(1-e^{-\kappa}\right), \\
& \widetilde{\alpha}=\alpha e^{-\kappa} .
\end{aligned}
$$

The parameter $\kappa$ is a measure of the correlations between barrier crossings and barrier fluctuations. In the case of the piecewise constant potential, the correlations
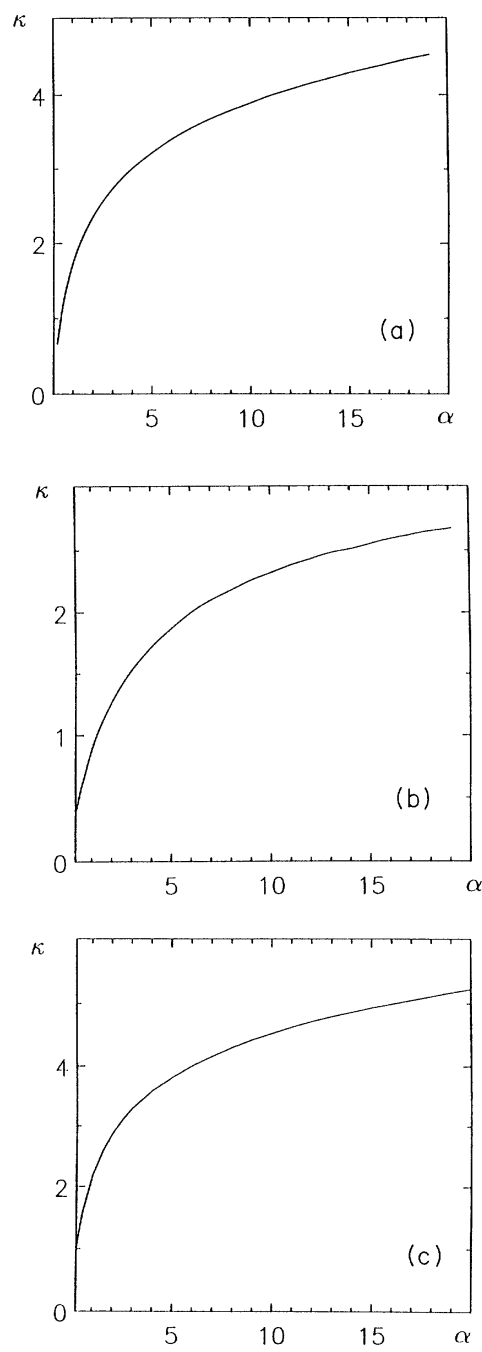

FIG. 4. The correlation parameter $\kappa$ as a function of the rate of the barrier fluctuations. (a) $E / T=10$ and $\eta=0.2$. (b) $E / T=5$ and $\eta=0.2$. (c) $E / T=10$ and $\eta=0.4$. are negligible,

$$
\kappa=0 \quad \text { [rectangular barrier] },
$$

whereas for the piecewise linear potential we have, cf. the discussion following Eq. (3.26),

$$
\left.\begin{array}{l}
\kappa \rightarrow \infty, \quad \alpha \rightarrow \infty \\
\kappa=0, \quad \alpha=0
\end{array}\right\} \quad \text { [linear barrier] }
$$

A more thorough understanding of the correlations between barrier crossings and barrier fluctuations is deferred to a subsequent publication. We have numerically calculated $\kappa$ as a function of the rate of the barrier fluctuations, $\kappa=\kappa(\alpha)$, for some values of the parameters $T$ and $\eta$, cf. Figs. 4(a) $-4(\mathrm{c})$. We observe first that $\kappa(\alpha)$ is a monotonical increasing convex function with $\kappa(0)=0$ and $\kappa \rightarrow \infty$ as $\alpha \rightarrow \infty$. A comparison of Figs. 4(a) and 4(b) shows that the correlations are enhanced as the temperature is lowered, i.e., $\kappa\left(\alpha, T_{a}\right)>\kappa\left(\alpha, T_{b}\right)$ for $T_{a}<T_{b}$. Further, Figs. 4(b) and 4(c) show that the correlations become stronger with increasing asymmetry $\eta=\left(E_{+}-E_{-}\right) /\left(E_{+}+E_{-}\right)$, i.e., $\kappa(\alpha, \eta)>\kappa\left(\alpha, \eta^{\prime}\right)$, for $\eta>\eta^{\prime}$

\section{SUMMARY AND CONCLUSIONS}

In this paper we have studied the thermally activated escape of an overdamped Brownian particle over a fluctuating potential barrier. We considered the case of a dichotomic random process: the barrier height fluctuates with a rate $\alpha$ between the values $E_{+}$and $E_{-}$. We started by first discussing the time scales of the escape of a Brownian particle over a stationary potential barrier. In this case, the mean exit time is proportional to the probability that the Brownian particle is thermally activated. We obtain the Arrhenius behavior, $\tau=\tau_{p} \exp (E / T)$, where $E$ is the energy difference between the top and the bottom of the barrier and $T$ is the temperature. The Arrhenius law depends on detailed properties of the Brownian motion in the potential well through the prefactor $\tau_{p}$ only. The splitting of the mean exit time into an exponential function and a prefactor has its physical origin in the separation of time scales. Thermally activated processes are slow on the time scales of the Brownian motion.

For the fluctuating barrier, we obtained exact results for the mean exit time for the piecewise linear and the piecewise constant potential barrier. We discussed in some detail the behavior of the mean exit time as a function of the rate of the barrier fluctuations.

In the case of the piecewise constant potential, the mean exit time is independent of the rate of the barrier fluctuations for both slow and fast barrier fluctuations, $\tau_{c} \sim \tau_{+}$for $\alpha<\tau_{+}^{-1}=\exp \left(-E_{+} / T\right)$ and $\tau_{c} \sim \tau_{-}$for $\alpha>\tau_{-}^{-1}=\exp \left(-E_{-} / T\right)$. In the intermediate regime, we find $\tau_{c} \sim \alpha^{-1}$ for $\tau_{+}^{-1}<\alpha<\tau_{-}^{-1}$.

For the piecewise linear potential, the mean exit time is qualitatively the same for slow and intermediate rates of the barrier fluctuations, i.e., $\tau_{l} \sim \tau_{+}$for $\alpha<\tau_{+}^{-1}$ and $\tau_{l} \sim \alpha^{-1}$ for $\tau_{+}^{-1}<\alpha<\tau_{-}^{-1}$. For fast barrier fluctuations, the mean exit time increases with increasing rates of the 
barrier fluctuations and approaches the asymptotic value, $\lim _{\alpha \rightarrow \infty} \tau_{l} \sim \sqrt{\tau_{+} \tau_{-}}=\exp \left[\left(E_{+}+E_{-}\right) / 2 T\right]$.

Hence, the "effective" activation energy, defined as $T \ln \tau$, depends on properties of the Brownian motion in the potential well. That is, for an arbitrary potential barrier, thermal activation processes and the Brownian motion are no longer separated in time. We discussed a simplified version of our model and showed that in the case of the piecewise constant potential, correlations between barrier crossings and barrier fluctuations are negligible. In the case of the piecewise linear potential, on the other hand, such correlations are important for fast barrier fluctuations.

We expand our results in several directions. Here, we briefly mention two of them. First, the Brownian motion in a general potential has properties similar to that in the piecewise linear and the piecewise constant potential. Correspondingly, the mean exit time for the general form of the potential barrier has similarities to both $\tau_{l}$ and $\tau_{c}$. In particular, we expect the correlation parameter $\kappa$ to depend on details of the potential barrier. It is interesting to investigate the behavior of $\kappa$ as we continuously transform the piecewise constant into the piecewise linear potential.

Second, our paper is restricted to the case of an overdamped Brownian particle. In this case, the three time scales $\tau_{ \pm}$and $\tau_{0}$ characterize the escape over the fluctuating piecewise linear potential barrier. If the energy of the Brownian particle dissipates with a finite rate, the damping constant defines still another time scale. It is thus important to extend our results to finite damping constants.

\section{ACKNOWLEDGMENTS}

U.Z. gratefully acknowledges the financial support from the Swiss National Science Foundation which made his stay at Clarkson University possible. C.R.D. acknowledges partial financial support from the NSF through Grant Nos. PHY-8958506, PHY-8907755, and PHY-9214715. We thank C. Van den Broeck for helpful comments.
*Current address: Theoret. Polymerphysik, Universität Freiburg, W-7800 Freiburg, Germany.

[1] H. Risken, The Fokker-Planck Equation (Springer-Verlag, Berlin, 1984).

[2] P. Hänggi, P. Talkner, and M. Borkovec, Rev. Mod. Phys. 62, 251 (1990).

[3] R. F. Fox, Phys. Rev. A 39, 4148 (1989).

[4] B. McNamara, K. Wiesenfeld, and R. Roy, Phys. Rev. Lett. 60, 2626 (1990).

[5] M. I. Dykman, R. Manella, P. V. E. McClintock, and N. G. Stocks, Phys. Rev. Lett. 65, 48 (1990); M. I. Dykman, P. V. E. McClintock, N. D. Stein, and N. G. Stocks, ibid. 67, 933 (1991).

[6] P. Jung and P. Hänggi, Phys. Rev. A 44, 8032 (1991).

[7] A. Bulsara, E. W. Jacobs, T. Zhou, F. Moss, and L. Kiss, J. Theor. Biol. 152, 531 (1991).

[8] V. I. Mel'nikov, Phys. Rev. A 45, 5474 (1992).
[9] D. L. Stein, R. G. Palmer, J. van Hemmen, and C. R. Doering, Phys. Lett. A 136, 353 (1989).

[10] D. L. Stein, C. R. Doering, R. G. Palmer, J. L. van Hemmen, and R. M. McLaughlin, J. Phys. A 23, L203 (1990).

[11] C. R. Doering and J. C. Gadoua, Phys. Rev. Lett. 69, 2318 (1992).

[12] V. Privman and H. L. Frisch, J. Chem. Phys. 94, 8216 (1991).

[13] C. W. Gardiner, Handbook of Stochastic Methods (Springer-Verlag, Berlin, 1985).

[14] P. Hänggi and P. Talkner, Phys. Rev. A 32, 1934 (1985).

[15] Rate constants that depend on the state of the random process have previously been used in, e.g., C. Van den Broeck and M. Bouten, J. Stat. Phys. 45, 1031 (1986).

[16] A similar stochastic model for resonant activation is studied in C. Van den Broeck (unpublished). 